Review

\title{
Recent Research and Development of Optical Fiber Monitoring in Communication Systems
}

\author{
Kunihiro TOGE and Fumihiko ITO* \\ NTT Access Network Service Systems Laboratories, NTT Corporation, Tsukuba, Ibaraki, 305-0805, Japan \\ *Corresponding author: Fumihiko ITO_E-mail: ito.fumihiko@lab.ntt.co.jp
}

\begin{abstract}
Recent progress on optical fiber monitoring in the optical communication systems is reviewed along with current optical fiber monitoring and diagnosing problems in deployed access, trunk and submarine communication systems.
\end{abstract}

Keywords: Optical fiber monitoring, diagnosis, PON monitoring, OFDR, OTDR

Citation: Kunihiro TOGE and Fumihiko ITO, "Recent Research and Development of Optical Fiber Monitoring in Communication Systems," Photonic Sensors, DOI: 10.1007/s13320-013-0127-2.

\section{Introduction}

Optical fiber networks began to be deployed for submarine and trunk systems in the 1980s in Japan to meet the demand for broadband communication. Fiber-to-the-home (FTTH) services were first introduced in 2001, and their provision to subscribers has spread rapidly over the access networks. The number of FTTH subscribers in Japan exceeded 23 million at the end of 2012 [1]. The number of subscribers is increasing, and a large number of optical fiber cables are being installed daily to meet this demand. Design, construction and maintenance technologies are of fundamental importance if we are to ensure the reliability of optical communication systems. For the efficient maintenance and operation of optical fiber networks, attention has been focused on optical fiber monitoring.

The basic functions of optical fiber monitoring in communication systems are designed to investigate for faults in optical fiber lines and to locate the fault with the appropriate resolution as a post-fault maintenance activity. The optical reflectometry is a promising technology for use in optical fiber monitoring in communication systems since operators can perform tests from central offices (or remotely via a network) without installing any equipment in the customer's house.

This paper focuses on the optical reflectometry and reviews optical fiber monitoring and applications that are deployed in current optical fiber networks. This paper also presents a review of recent developments in optical fiber monitoring within our group, along with the diagnosing problems with access, trunk and submarine fiber networks.

\section{Optical fiber monitoring in current optical fiber cable networks}

In this section, we review optical fiber monitoring based on the optical time domain reflectometry (OTDR) and related configurations deployed in current optical access, trunk and

Received: 16 July 2013 / Revised version: 27 August 2013

(C) The Author(s) 2013. This article is published with open access at Springerlink.com 
submarine cable networks.

\subsection{Access network}

An optical fiber line testing system has been deployed to support the construction, reconfiguration, and maintenance of a large number of optical fiber lines in access networks [2, 3]. It performs various types of the optical test remotely, and its functions are shown in Table 1. Figure 1 shows the configuration of an optical fiber line system used in optical access networks. An operator in a maintenance center sends orders via a data network to the optical testing module (OTM) telling it to perform various tests. The OTM, which is installed in an optical distribution frame (ODF), contains various types of optical fiber measurement equipments such as an OTDR unit, a light source for optical fiber identification, and a power meter. An optical switch called a fiber selector selects the target fiber, and an optical coupler introduces the test light into the target fiber. During construction work, OTDR test is performed to measure the loss and reflectance at splice points with a typical resolution of around twenty meters. When responding to breakdowns or complaints, it is used to distinguish faults between the transmission equipment and an optical fiber line and to identify the fault location.

Table 1 Functions of optical fiber line testing system.

\begin{tabular}{c|c|l}
\hline Scene & \multicolumn{2}{|c}{ Function } \\
\hline $\begin{array}{c}\text { Construction } \\
\text { Reconfiguration }\end{array}$ & OTDR test & Connection loss and reflection \\
\cline { 2 - 3 } Maintenance & Loss test & Optical cable span loss \\
\hline \multirow{2}{*}{ Fault test } & $\begin{array}{l}\text { Fault isolation } \\
\text { Fault location }\end{array}$ \\
\cline { 2 - 3 } & Periodic test & Detection of water penetration \\
\hline Identification & Insertion of identification light \\
\hline
\end{tabular}

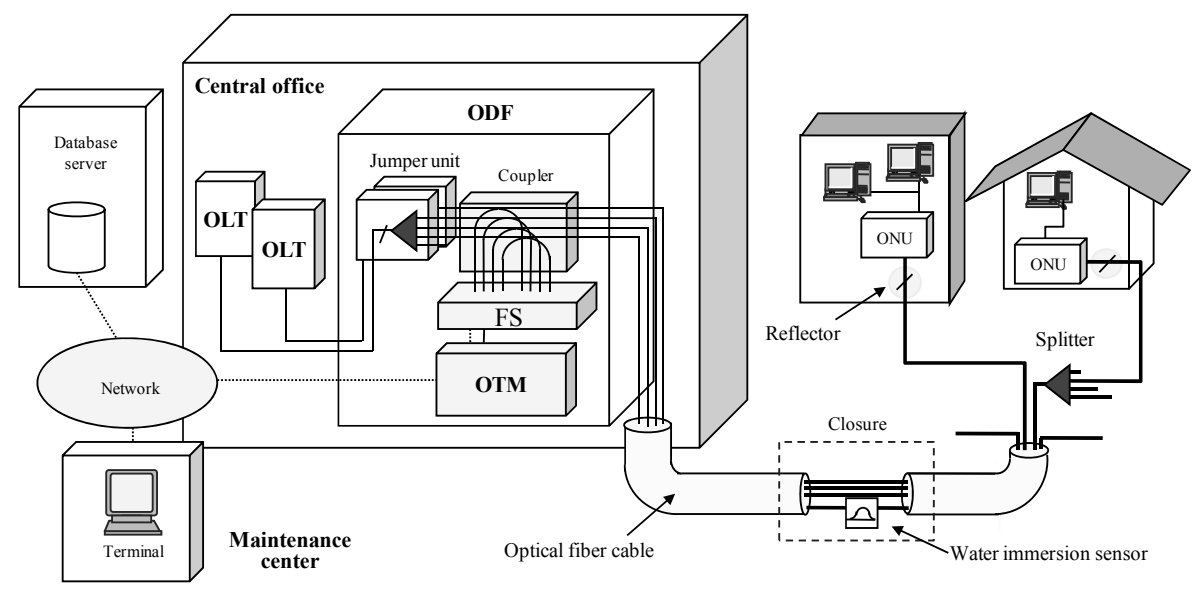

Fig. 1 Schematic configuration of the optical fiber line system for access networks.

A reflector installed in front of an optical network unit (ONU) at a subscriber's house cuts and reflects the test light from the OTM while allowing the communication light from the optical line terminal (OLT) to pass. A fiber Bragg grating, which has a sharp cutoff characteristic and high reflectivity, is used for the reflector. Therefore, we can carry out in-service testing without affecting the transmission quality. The wavelength of the test light is $1650 \mathrm{~nm}$, which is sensitive to the fiber bending loss, in compliance with ITU-T L.41 [4].

\subsection{Trunk network}

Optical trunk networks are important communication lines between central offices and have a typical span length of up to about $80 \mathrm{~km}$. The typical configuration of an optical trunk line is shown in Fig. 2. It differs from an optical access network in that there are fewer fibers in the cables, and it uses a pair of fibers as upstream and downstream transmission lines. In general, optical trunk lines are relatively low loss and extend over long distances compared with optical access 
networks. The OTDR is also used for testing after construction, fault and periodic testing with a typical resolution of several hundred meters. When the link loss of an optical trunk line exceeds the dynamic range of the OTDR, bidirectional measurement is carried out from both ends of the optical trunk line.

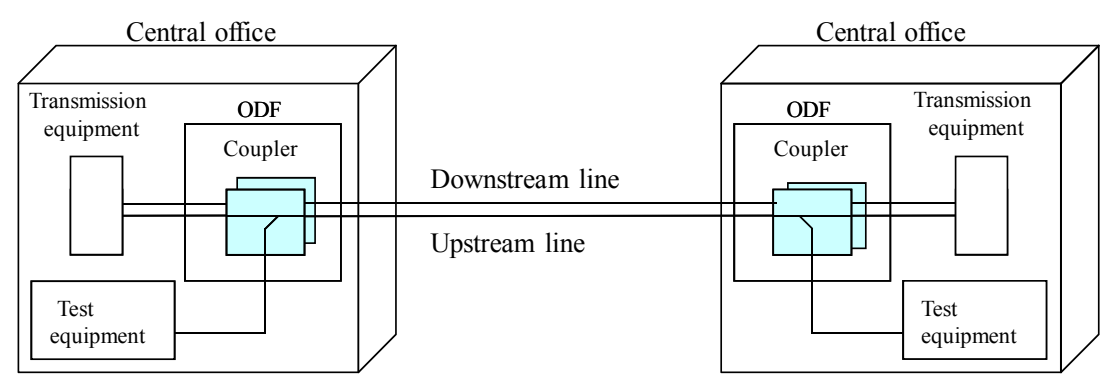

Fig. 2 Typical optical trunk line monitoring configuration.

Since optical trunk lines, which accommodate, for example, dense wavelength division multiplexing (DWDM) and coarse WDM (CWDM) transmissions, may carry a high total optical power, several technical considerations are required as regards nonlinear effects such as spontaneous Raman scattering in relation to in-service testing [5].

In accordance with the requirements of the transmission equipment, spectral attenuation and dispersion characteristics such as chromatic dispersion and polarization mode dispersion (PMD) attributed to existing optical fiber cables are occasionally verified when the transmission systems are developed.

\subsection{Submarine network}

Optical submarine cable systems are divided into two categories. One category consists of repeatered systems for long-haul applications, which transmit signals over thousands of kilometers by using optical amplifiers [erbium doped fiber amplifiers (EDFAs)] and power fed through submarine cables. The other category consists of repeaterless systems for short-distance applications of up to several hundred kilometers.

To monitor the long submarine optical fiber cable, a coherent OTDR (C-OTDR) is employed to improve the receiver sensitivity up to the quantum limit by heterodyne detection, which is advantageous for the direct detection. A typical resolution of over one kilometer is used for submarine applications. Roughly speaking, a repearterless system has basically the same monitoring aspect as that in optical trunk networks. In repeatered systems, the EDFA contains optical isolators and auto-level control (ALC) circuits to

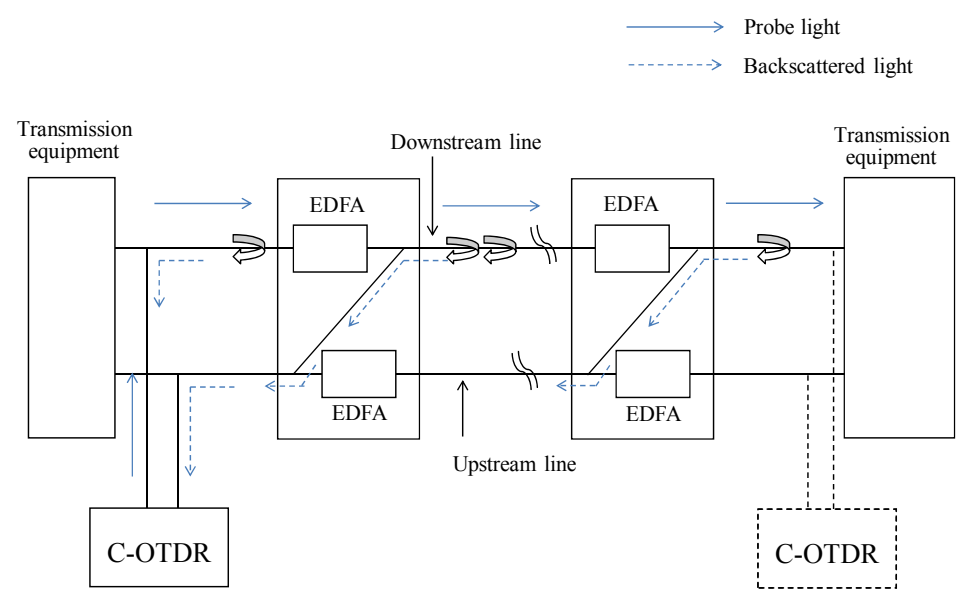

Fig. 3 Typical monitoring configuration for the repeatered submarine transmission line. 
stabilize the gain characteristic of communication signals passing through the repeaters. Figure 3 shows a typical monitoring configuration for repeatered submarine systems [6]. Since the optical isolator rejects any backscattering light from the second and subsequent spans of the transmission line, an optical coupler with a typical coupling ratio of 1:10 connects the downstream and upstream lines. The backscattered light generated in the second and subsequent spans of the downstream line propagates back along the upstream line via the backscattered paths incorporated in each repeater and then detected in the C-OTDR. The repeater gain, fiber loss, and fiber fault location are monitoring parameters [7].

\section{Recent research on optical fiber monitoring in communication systems}

\subsection{End-reflection assisted Brillouin analysis for PON branch monitoring}

\section{A. Issues with current monitoring in access networks}

There is one of difficulties to maintain the passive optical network (PON) widely installed in access networks, in which an optical power splitter is installed outside. The simple OTDR approach at a central office provides only a superposition of the reflectometric traces of all branches. The high resolution OTDR is one of the few practical ways in the current situation whereby operators can confirm the number of reflections at the FBG reflector in the traces, but its use is limited to checking for the presence of fiber breaks [8]. Otherwise, operators have to undertake testing from inside the customer's house [9].

The above problem is widely recognized as a research topic of interest in optical fiber monitoring. Many approaches have been reported including the multi-wavelength OTDR with wavelength dependent devices [10,11] and Brillouin OTDR with devices that have different Brillouin frequency shifts [12]. However, these techniques require additional optical components or changes to the optical fiber itself. Therefore, they are difficult to apply to existing PONs.

Recently, we have proposed and developed a technique to overcome this problem, namely to enable us to monitor the individual loss distribution of PON branches from the central office for the configuration shown in Fig. 2 without any change in the installed facilities $[13,14]$. We call this approach end-reflection assisted Brillouin time domain analysis. The next subsection presents a review of recent achievements regarding this technique including the measurement of a 32-branched PON, with a dynamic range of about $25 \mathrm{~dB}$.

\section{B. End-reflection assisted Brillouin analysis}

Figure 4 shows the principle of end-reflection

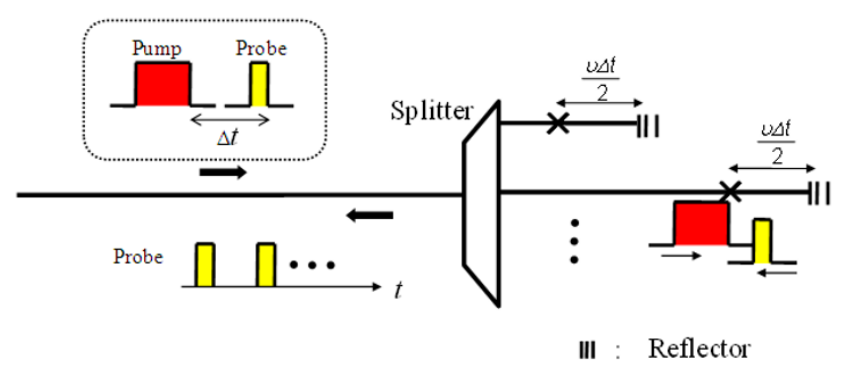

Fig. 4 Principle of end-reflection assisted Brillouin analysis.

assisted Brillouin analysis. The test beam consists of a probe pulse accompanied by a pump pulse with a temporal interval of $\Delta t$. Their optical frequencies are set such that a Brillouin interaction occurs, and the probe pulse is amplified when the two pulses collide at a distance of $v \Delta t / 2$ from the reflection point at the end of the optical fibers ( $v$ : light velocity). Since the Brillouin gain is proportional to the pump power at the collision, the optical loss at the collision point in every branch can be obtained by observing the probe pulse gain caused by the interaction. To distinguish the branches requires the difference in the lengths of the branches, namely, a length difference that exceeds the probe pulse width is needed between every pair. In other words, when a small length difference is included in the measured PON, a short probe pulse must be used. Consequently, a large 
bandwidth is needed, and the sensitivity is decreased. The length difference (and probe pulse width) should be larger than the lifetime of an acoustic phonon, otherwise the gains of two branches with similar lengths cannot be distinguished. The event spatial resolution of the system is determined by the smaller of the two values, namely the pump and probe pulse widths. A larger pump pulse width yields a larger gain, thus improving the sensitivity. In a laboratory test of the described method, where a PON test-bed was constructed by using standard single-mode fibers (SSMFs) and optical couplers, we demonstrated the measurement of a 32-branched PON with a minimum branch length difference of $2 \mathrm{~m}$. The loss distribution of every branch was successfully obtained. Figure 5 shows the measured loss distribution at $1.55 \mu \mathrm{m}$ and provides the results for eight of the 32 branches. Figures 5(b) and 5(g) show the losses that were intentionally added to the branches.

The signal-to-noise ratio (SNR), or dynamic range of the measurement system was analyzed and examined experimentally. We found that the sensitivity of the measurement was mainly determined by the receiver noise, which was combined with the probe beam when analyzing the Brillouin gain. However, in some cases, the spontaneous Brillouin scattering yielded at all the branches and combined at the receiver may exceed the receiver noise and be a limiting factor as regards the sensitivity. In a laboratory test with a minimum length difference of $2 \mathrm{~m}$, an event resolution of $10 \mathrm{~m}$, an input pump power of $+16 \mathrm{dBm}$, and assuming a uniform Brillouin frequency shift (BFS) value throughout the fiber, we obtained a dynamic range of about $25 \mathrm{~dB}$ with 10000-time averaging (1 2 minutes measurement time).

Further investigation is now under way to respond the BFS variation in the field, maintenance wavelength for in-service testing, intended for the practical use in optical access networks.
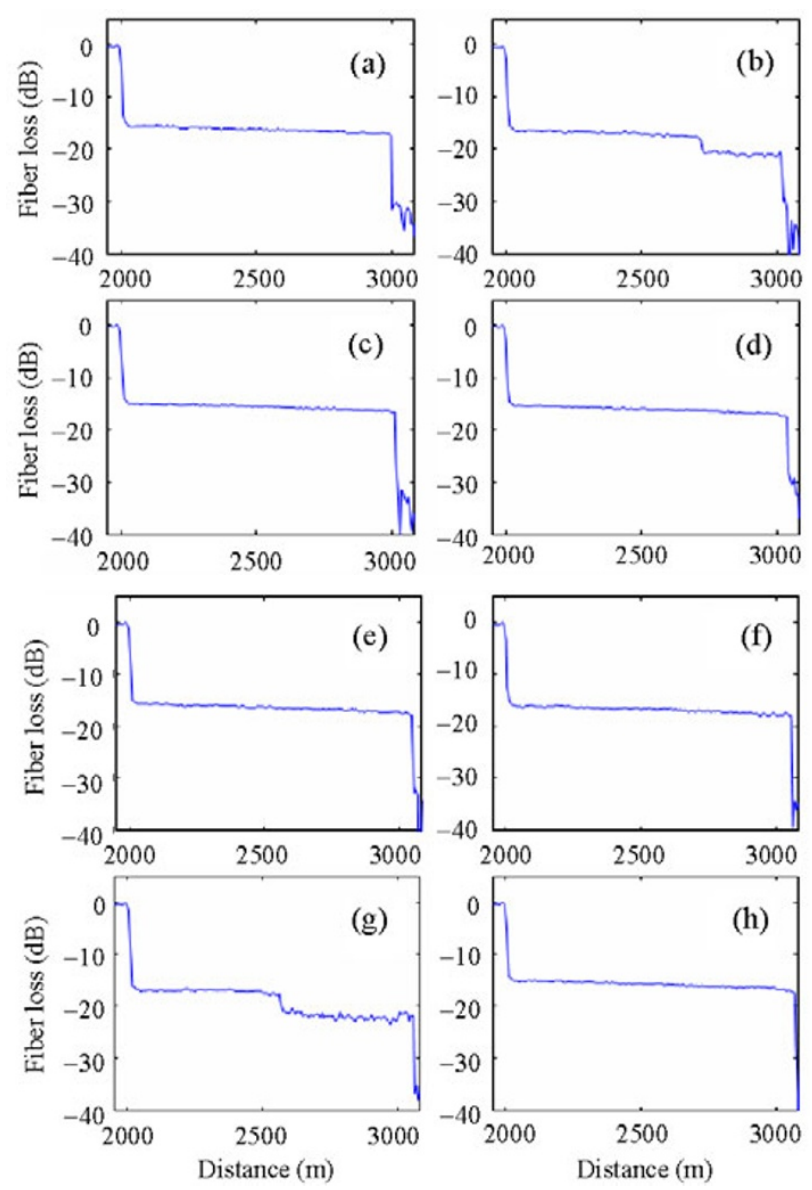

Fig. 5 Example of measured loss distribution in eight of the 32 branches (the measured wavelength was $1.55 \mu \mathrm{m}$ ).

\subsection{Long-range C-OFDR for identifying high-PMD section along installed cables}

\section{A. Issue with legacy cables in trunk networks}

The PMD of the optical fiber is an important factor when designing large capacity transmission systems. In particular, legacy fibers installed in trunk networks prior to the early 1990s often exhibit high PMD values that are unsuitable for high-speed transmission. Although the digital coherent technology significantly mitigates the stringent requirement for decreasing PMD, in terms of fiber management, it is very useful to know the distribution of such a large birefringence along all installed fibers.

The polarization-sensitive optical time domain reflectometry (P-OTDR) [15] is a useful tool for measuring the states of polarization (SOP) of 
backscattered lightwaves, from which we can know the beat length. Techniques for calculating birefringence, or even PMD, by employing the backscattered SOP measured with the P-OTDR, have progressed greatly [16-20]. However, the spatial resolution of the P-OTDR is limited to about $1 \mathrm{~m}$, and this is often insufficient to recognize the short beat length induced by a high birefringence.

The coherent optical frequency domain reflectometry (C-OFDR) provides a narrow spatial resolution for detecting the birefringence of highPMD fibers [21, 22]. However, its measurement range is limited by the coherence length of the optical source. To maintain a narrow spatial resolution while extending the measurement range, we have proposed and developed the phase-noisecompensated OFDR (PNC-OFDR) [23] and obtained a sub-cm spatial resolution over $40 \mathrm{~km}$ in a normal laboratory environment [24].

In this subsection, we describe the application of the PNC-OFDR to locate sections with high birefringence along installed optical cables. We found that an installed fiber occasionally included polarization beat lengths as short as a few tens of $\mathrm{cm}$, which are very difficult to observe with the P-OTDR.

B. Phase noise compensated C-OFDR (PNC$O F D R)$ and distributed birefringence measurement [23-26]

Figure 6 shows the configuration and principle of the PNC-OFDR. The setup consists of two interferometers: a measurement interferometer (MI) and a reference interferometer (RI). The probe beam is divided into two equal portions, which are simultaneously incident to the MI and RI. The MI collects backscattered beam data from the fiber under test (FUT) as in conventional C-OFDR. The beat signal obtained from the MI, $b(t)$, corresponding to the reflection at the roundtrip time $\tau$, is given by

$$
b(t)=\cos [\pi \gamma \tau t+\phi(t)-\phi(t-\tau)]
$$

where $\gamma$ is the swept rate of the frequency, and $\phi(t)$ represents the phase noise. The PNC-OFDR compensates for the beat fluctuation that originates from the phase noise of the laser with the help of the RI. The RI is a Mach-Zehnder interferometer with a delay $\tau_{\text {ref }}$ and originates a reference beat signal given by

$$
r(t)=\cos \left[\pi \gamma \tau_{\text {ref }} t+\phi(t)-\phi\left(t-\tau_{\text {ref }}\right)\right] .
$$

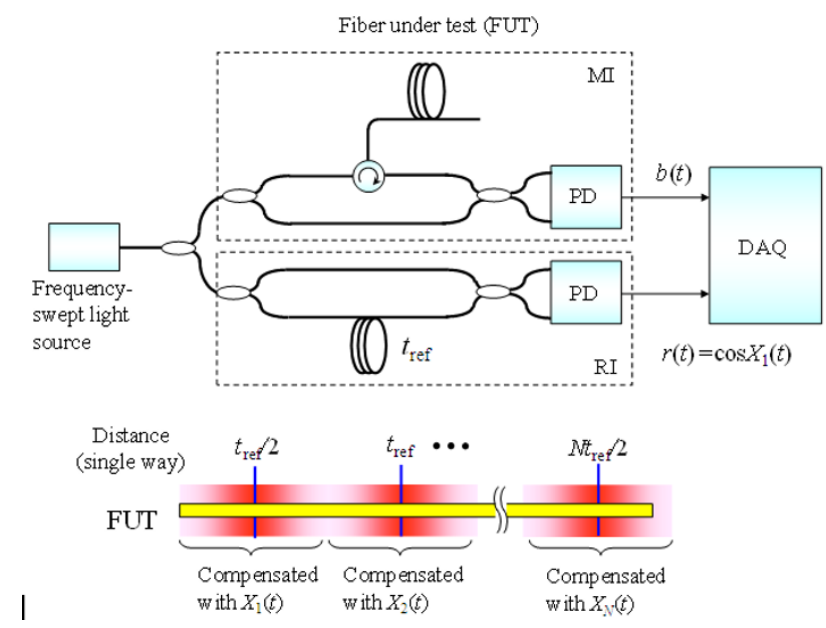

Fig. 6 Schematic configuration and principle of PNC-OFDR.

When the (roundtrip) measurement distance equals the reference delay, the beat frequency fluctuation of the RI is in accordance with the beat frequency to be measured in the MI, therefore, the beat frequency fluctuation (or phase noise) can be compensated by using the reference beat signal directly. However, this is not true when the measured distance is far from the reference delay. When the inside of the bracket in (2) is replaced by $X_{1}(t)$, we define and consider a new reference signal as follows:

$$
X_{N}(t)=\sum_{n=0}^{N-1} X_{1}\left(t-n \tau_{\text {ref }}\right) .
$$

Accordingly, the $N$-th generated reference is given by

$$
X_{N}(t)=N \pi \gamma \tau_{\text {ref }} t+\phi(t)-\phi\left(t-N \tau_{\text {ref }}\right) .
$$

Equation (4) indicates that $X_{N}(t)$ is the reference signal corresponding to the distance $N \tau_{\text {ref }}$, which is effective at a distance of integer times the reference delay. Even if the distance does not match $N \tau_{\text {ref }}$ 
exactly, $X_{N}(t)$ is effective for the difference within the coherence length. We confirmed that a reference signal generated in this way works well even at distances of 16 to 32 times the reference delay, or the coherence length of the light source.

With PNC- (or conventional C-) OFDR with dual polarization receivers, we can observe the evolution of the backscattered SOP throughout the propagation $[21,22]$. The evolution of the SOP through propagation in birefringent media is described by the dynamical equation:

$$
\frac{d s}{d z}=\beta(z) \times s(z)
$$

where $\beta=\left(\beta_{1}, \beta_{2}, \beta_{3}\right)$ is the birefringent vector, and $s$ is the Stokes vector of the SOP. The evolution of the backscattered SOP is also described by the same equation, with the roundtrip birefringence vector, $\beta_{R}(z)$, which is related to the forward birefringence vector by

$$
\beta_{R}(z)=2 \beta_{L}(z)=\left(2 \beta_{1}, 2 \beta_{2}, 0\right)
$$

where $\beta_{L}(z)=\left(\beta_{1}, \beta_{2}, 0\right)$ is the linear birefringence vector. In the following experiments designed to analyze local linear birefringence (and missing the circular birefringence term), we used this simple and useful relationship found in [17].

The results of two examples are shown in Fig. 7. Here, columns are used to show the locations of the splice points, which are introduced from a cable management database. To suppress the speckle noise

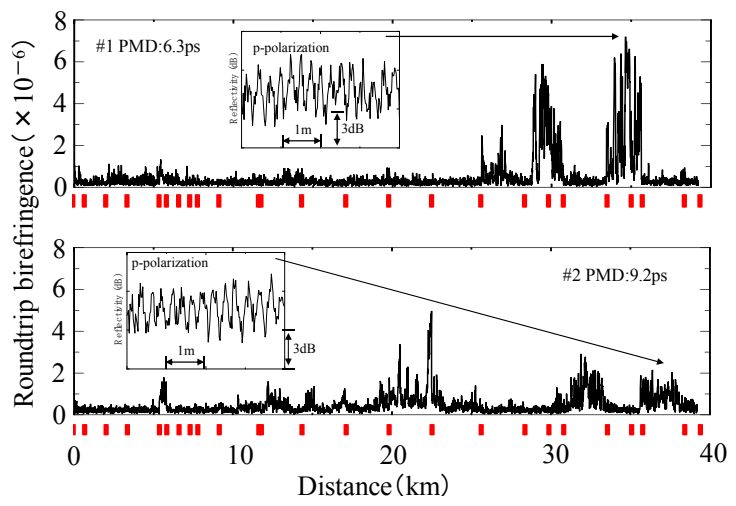

Fig. 7 Two examples of distributed linear birefringence measurement (the insets show the p-polarization reflectivity of the PNC-OFDR in the high-birefringence section). of the Rayleigh scattering, the measurement was performed and incoherently averaged within a wavelength range of $0.4 \mathrm{~nm}$. It is clear that the round-trip birefringence was not uniform along the fiber, and several sections had large values. Abrupt changes in the round-trip birefringence were observed at certain splice points, confirming the validity of our measurement, since different kinds of fibers may be spliced in the cable. The insets in Fig. 7 give the p-polarization reflectivity of the high-birefringence section. It is seen that the beat lengths are very short in the sections where the birefringence is high. One part even exhibits a beat length of only about $20 \mathrm{~cm}$, which could not be detected by the conventional P-OTDR, or, to the best of our knowledge, by any other type of the reflectometry over such a long range.

\subsection{Frequency division multiplexing coherent OTDR for submarine cable monitoring}

A. Issues with construction and maintenance of submarine optical fiber cables

As mentioned in Section 2.3, the C-OTDR is used for fault location and characterization in long-haul submarine applications. In the repeatered system shown in Fig. 3, repeater spans occasionally exceed $100 \mathrm{~km}$. There is a problem in that the dynamic range (DR) is sometimes insufficient, because the peak power of the probe pulse from the repeater is restricted by the output power of the EDFA in the repeaters.

To achieve a good signal-to-noise ratio in reflectometry traces, a large number of measurements must be performed for averaging. Manufactured submarine transmission lines (including optical cables, repeaters, etc.) are examined under several circumstances, for example during acceptance inspection, shipping, final inspection after laying and when identifying a fiber fault during operation. There has been a need for a more sensitive reflectometry technique to shorten the measurement time. 
An approach employing a coded pulse train has been reported as a technique for improving the sensitivity while retaining the same spatial resolution [27, 28]. A pulse train that has an orthogonal code with a time shift provides theoretically the distributed reflectivity along the optical fiber by auto-correlation. However, this approach may induce large crosstalk effects owing to insufficient side lobe suppression since it is not easy for repeatered systems that incorporate a large gain exceeding several decades of decibels in optical amplifiers. On the other hand, another approach has been reported in which the optical frequency of the probe pulse is encoded [29]. We used the latter approach as a basis, since it is easy to suppress side lobes by employing a sufficient narrow band laser as a light source, and we developed a new C-OTDR technique. This approach requires a broad receiver bandwidth compared with the code pulse train technique, but it is easy to obtain the bandwidth with a recently developed high-speed balanced detector and an analog to digital (A/D) converter with several hundreds of MHz. We call this C-OTDR approach frequency division multiplexing OTDR (FDM-OTDR).

\section{B. High sensitivity measurement by FDM-OTDR} [30]

Figure 8 shows the basic concept of our FDM-OTDR. A probe pulse with multiple frequencies is launched into the FUT. Each frequency component is serially arranged in the time domain thus avoiding both a peak power increase and a nonlinear interaction as a result of four-wave mixing. They are detected by a single photodetector, which is responsible for the total test light pulse bandwidth, and digitized by an A/D converter. The digitized backscattered signals can be separated into individual frequency components with a fast Fourier transform (FFT). As a result, multiple OTDR traces measured at different frequencies can be obtained simultaneously. This means that several C-OTDR traces can be obtained within a single iteration of the measurement. Hence, the DR per iteration can be improved by using this scheme. The round-trip DR improvement in the FDM-OTDR is given by

$$
10 \lg \sqrt{N_{\text {FDM }}}(\mathrm{dB})
$$

where $N_{\mathrm{FDM}}$ is the number of frequencies in the probe pulse. Frequency demultiplexing is performed based on software processing, which indicates that no specific hardware is needed for demultiplexing, and it is not much more complicated than the conventional C-OTDR.

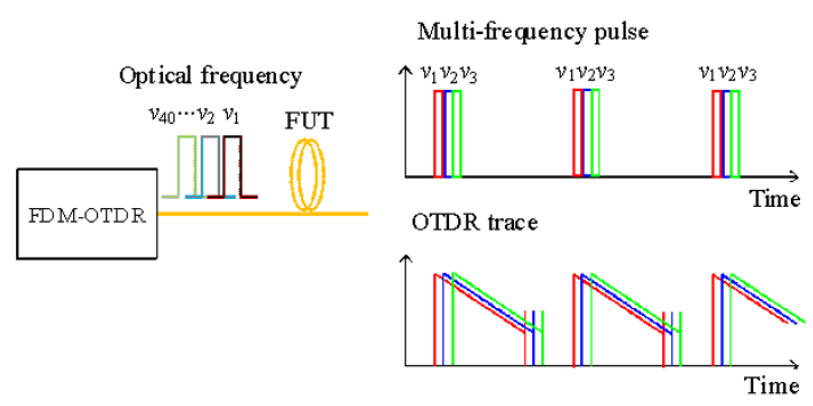

Fig. 8 Basic concept of the FDM-OTDR.

Figure 9 shows OTDR traces that were obtained

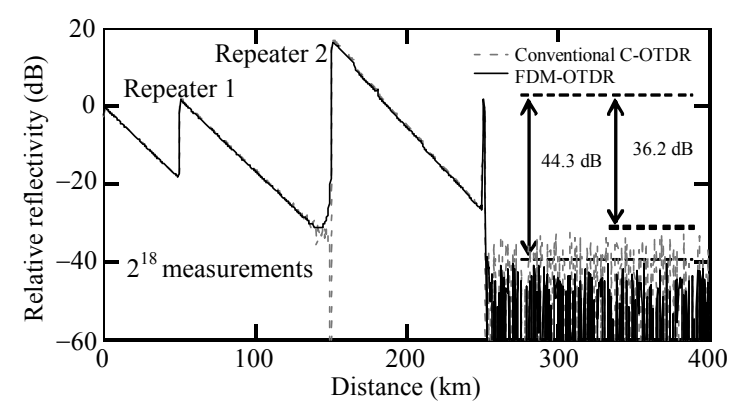

Fig. 9 OTDR traces obtained by FDM-OTDR and conventional C-OTDR, respectively.

with the conventional C-OTDR and FDM-OTDR with $2^{18}$ measurements. The $N_{\mathrm{FDM}}$ and the frequency spacing were $40 \mathrm{kHz}$ and $800 \mathrm{kHz}$, respectively. The observed DRs were $36.2 \mathrm{~dB}$ and $44.3 \mathrm{~dB}$. Here, the DR was the round-trip DR and was defined by the ratio between the near-end Rayleigh level and the RMS noise level. The FDM-OTDR obtained an 8.1-dB higher DR than the conventional C-OTDR. The calculated result obtained using (7) was about $8 \mathrm{~dB}$ and agreed well with the experimental results. It is seen that the shapes of the OTDR trace in front of the repeaters, in which the reflectivity is 
precipitously changed, exhibit almost no broadening. This indicates that the inter-channel crosstalk of the Rayleigh backscattered light was sufficiently suppressed in our FDM-OTDR.

The software-based processing in the FDM-OTDR benefited from recent increases in the capacity of computer processors. We noted that the FDM-OTDR required 1.3 times the pulse repetition period to finish the calculation during a pulse round trip, which corresponded to an SNR degradation of less than $0.6 \mathrm{~dB}$. This can be minimized by the high-speed implementation of an FFT. We expect the FDM-OTDR to be deployed for practical applications in the near future since a short measurement time is advantageous with respect to reducing the construction and operational costs of submarine networks.

\section{Conclusions}

Recent research and development in relation to optical fiber monitoring were reviewed. The optical reflectometry is a promising technology that enables operators to maintain a large number of facilities. There is no doubt that simple OTDR (or C-OTDR) approaches play a role as the main technology for current optical fiber monitoring in communication systems. However, the diagnosing problems in communication systems will become more multifaceted as the systems progress and will require different functions related to optical access, trunk and submarine configurations and applications. Further research and development of optical fiber monitoring technologies are expected with a view to realizing highly reliable optical services.

\section{Acknowledgment}

We thank Y. Azuma for his continuous support.

Open Access This article is distributed under the terms of the Creative Commons Attribution License which permits any use, distribution, and reproduction in any medium, provided the original author(s) and source are credited.

\section{References}

[1] Ministry of Internal Affairs and Communications in Japan, "Information and communications statistics and database," 2012.

[2] M. Arii, Y. Azuma, Y. Enomoto, K. Suzuki, N. Araki, S. Uruno, et al., "Optical fiber network operation technologies for expanding optical access network services," NTT Technical Review, vol. 5, no. 2, pp. 32-38, 2007.

[3] Y. Enomoto, H. Izumita, K. Mine, and N. Tomita, "Design and performance of novel optical fiber distribution and management system with testing functions in central office," Journal of Lightwave Technology, vol. 29, no. 12, pp. 1818-1834, 2011.

[4] ITU-T Recommendation L.41, "Maintenance wavelength on fibres carrying signals," 2000.

[5] K. C. Reichmann, N. J. Frigo, and X. Zhou, "In-service OTDR limitations in CWDM systems caused by spontaneous Stokes and anti-Stokes Raman scattering," IEEE Photonics Technology Letters, vol. 16, no. 7, pp. 1787-1789 2004.

[6] S. Furukawa, K. Tanaka, Y. Koyamada, and M. Sumida, "Enhanced coherent OTDR for long span optical transmission lines containing optical fiber amplifiers," IEEE Photonics Technology Letters, vol. 7, no. 5, pp. 540-542, 1995.

[7] ITU-T Recommendation G.979, "Characteristics of monitoring systems for optical submarine cable systems," 2012.

[8] Y. Enomoto, H. Izumita, and M. Nakamura, "Over $31.5 \mathrm{~dB}$ dynamic range optical fiber line testing system with optical fiber fault isolation function for 32-branched PON," in Proc. Optical Fiber Communication Conference 2003 (OFC2003), Mar. 23-28, vol. 2, pp. 608-609, 2011.

[9] Y. Koshikiya, N. Araki, H. Izumita, and F. Ito, "Simple and cost-effective fault location technique using bi-directional OTDR and in-service line testing criteria for PONs," in Proc. European Conference on Communications 2005 (ECOC2005), Sep. 25-29, vol. 1, pp. 83-84, 2005.

[10] K. Tanaka, M. Tateda, and Y. Inoue, "Measuring the individual attenuation distribution of passive branched optical networks," IEEE Photonics Technology Letters, vol. 8, no. 7, pp. 915-917, 1996.

[11] U. Hilbk, M. Burmeister, B. Hoen, T. Hermes, J. Saniter, and F. Westphal, "Selective OTDR measurements at the central office of individual fiber links in a PON," presented at Proc. Conference on Optical Fiber Communication (OFC 97), Dallas, America, Feb. 16-21, 1997. 
[12] D. Iida, N. Honda, H. Izumita, and F. Ito, "Design of identification fibers with individually assigned Brillouin frequency shifts for monitoring passive optical networks," Journal of Lightwave Technology, vol. 25, no. 5, pp. 1290-1297, 2007.

[13] F. Ito, H. Takahashi, and K. Toge, "End-reflection assisted brillouin measurement for PON monitoring," presented at Proc. CLEO-PR\&OECC/PS 2013, MS2-1, 2013.

[14] H. Takahashi, F. Ito, C. Kito, and K. Toge, "Individual loss distribution measurement in 32-blanched PON using pulsed pump-probe Brillouin analysis," Optics Express, vol. 21, no. 6, pp. 6739-6748, 2013.

[15] A. J. Rogers, "Polarization-optical time domain reflectometry: a technique for the measurement of field distributions," Applied Optics, vol. 20, no. 6, pp. 1060-1074, 1981.

[16] J. G. Ellison and A. S. Siddiqui, "Automatic matrix-based analysis method for extraction of optical fiber parameters from polarimetric optical time domain reflectometry data," Journal of Lightwave Technology, vol. 18, no. 9, pp. 1226-1232, 2000.

[17] A. Galtarossa and L. Palmieri, "Spatially resolved PMD measurements," Journal of Lightwave Technology, vol. 22, no. 4, pp. 1103-1115, 2004.

[18] R. Goto, S. Tanigawa, S. Matsuo, and K. Himeno, "On-spool PMD estimation method for low-PMD fibers with high repeatability by local-DGD measurement using POTDR," Journal of Lightwave Technology, vol. 24, no. 11, pp. 3914-3919, 2006.

[19] H. Dong, P. Shum, J. Q. Zhou, G. X. Ning, Y. D. Gong, and C. Q. Wu, "Spectral-resolved backreflection measurement of polarization mode dispersion in optical fibers," Optics Letters, vol. 32, no. 12, pp. 1665-1667, 2007.

[20] D. Fritzsche, M. Paul, L. Schuerer, A. Ehrhardt, D. Breuer, W. Weiershausen, et al., "Measuring the link distribution of PMD: field trial using an RS-POTDR," presented at Proc. of OFC/NFOEC 2008, San Diego, California, America, Feb. 24-28, 2008.

[21] B. Huttner, J. Reecht, N. Gisin, R. Passy, and J. P. Weid, "Local birefringence measurements in single-mode fibers with coherent optical frequency-domain reflectometry," IEEE Photonics Technology Letters, vol. 10, no. 10, pp.1458-1460, 1998.

[22] M. Wegmuller, M. Legre, and N. Gisin, "Distributed beatlength measurement in single-mode fibers with optical frequency-domain reflectometry," Journal of Lightwave Technology, vol. 20, no. 5, pp. 800-807, 2002.

[23] F. Ito, X. Fan, and Y. Koshikiya, "Long-range coherent OFDR with light source phase noise compensation," Journal of Lightwave Technology, vol. 30, no. 8, pp. 1015-1024, 2012.

[24] X. Fan, Y. Koshikiya, and F. Ito, "Phasenoise-compensated optical frequency domain reflectometry," IEEE Journal of Quantum Electronics, vol. 45, no. 6, pp. 594-602, 2009.

[25] X. Fan, Y. Koshikiya, and F. Ito, "Centimeter-level spatial resolution over $40 \mathrm{~km}$ realized by bandwidth-division phase-noise-compensated OFDR," Optics Express, vol. 19, no. 20, pp. 19122-19128, 2011.

[26] F. Ito, Y. Koshikiya, and X. Fan, "Identification of high-PMD sections along installed optical cables with long range OFDR," in Proc. Optical Fiber Communication Conference (OFC2013), Anaheim, America, Mar. 17-21, pp. 1-3, 2013.

[27] K. Okada, K. Hashimoto, T. Shibata, and Y. Nagaki, "Optical cable fault location using correlation technique," Electronics Letters, vol. 16, no. 16, pp. 629-630, 1980.

[28] M. Nazarathy, S. A. Newton, R. P. Giffard, D. S. Moberly, F. Sischka, W. R. Trutna, et al., "Real-time long range complementary correlation optical time domain reflectometer," Journal of Lightwave Technology, vol. 7, no. 1, pp. 24-38, 1989.

[29] M. Sumida, "Optical time domain reflectometry using an M-ary FSK probe and coherent detection," IEEE/OSA Journal Lightwave Technology, vol. 14, no. 11 , pp. 2483-2491, 1996.

[30] H. Iida, Y. Koshikiya, F. Ito, and K. Tanaka, "Ultra high sensitive coherent optical time domain reflectometry employing frequency division multiplexing," Journal of Lightwave Technology, vol. 30, no. 8, pp. 1121-1126, 2012. 